\title{
Factors associated with mortality among patients with active pulmonary tuberculosis requiring intensive care
}

\author{
Wann Jia $\underline{L O h}^{1}$, MBBS, MRCP, Yue $\underline{Y u}^{2}$, Chian Min $\underline{L O O}^{3}$, MRCP, FAMS, Su Ying $\underline{L} \underline{O W}^{3}$, BMBCh, MRCP
}

INTRODUCTION The aim of this study was to identify patient characteristics and risk factors associated with in-hospital mortality of patients with pulmonary tuberculosis (PTB) requiring intensive care unit (ICU) management.

METHODS A retrospective chart review was conducted of all patients with active PTB admitted to the ICU at Singapore General Hospital, Singapore, between January 2005 and December 2010.

RESULTS There were 2,155 patients with active PTB diagnosed, of whom 83 (3.9\%) patients were admitted to the ICU, but eight were excluded because their admission to the ICU was unrelated to PTB. The most common comorbidities were diabetes mellitus $(n=23,30.7 \%)$ and immunocompromised host $(n=25,33.3 \%)$. A few $(n=4,5.3 \%)$ of the patients had HIV coinfection. A majority $(n=67,89.3 \%)$ of patients required mechanical ventilation and the mean duration of mechanical ventilation was $8.05 \pm 14.43$ days. Mean duration of ICU stay and hospital stay were $10.23 \pm 15.8$ days and $33.7 \pm 50.7$ days, respectively. In-hospital mortality was $62.7 \%(n=47)$, and 36 of these patients died while in the ICU (ICU mortality, 48.0\%). Univariate analysis identified ischaemic heart disease, low albumin, Acute Physiology and Chronic Health Evaluation score, disseminated intravascular coagulation, shock and multiorgan failure as significantly associated with mortality. Multivariate analysis showed that low albumin on the day of ICU admission was the only significant independent predictor of death $(p=0.033)$.

CONCLUSION In-hospital mortality from active PTB requiring ICU admission was $62.7 \%$, and low albumin was an independent predictor of mortality in this study.

Keywords: acute respiratory distress syndrome, human immunodeficiency virus, intensive care unit, pulmonary tuberculosis

\section{INTRODUCTION}

Pulmonary tuberculosis (PTB) is a worldwide public health problem and the second greatest infectious disease killer after the human immunodeficiency virus (HIV). ${ }^{(1)}$ The incidence of tuberculosis (TB) in Singapore was 36.9 per 100,000 population in 2013. ${ }^{(2)}$ A majority of patients had PTB with or without extrapulmonary involvement $(86.3 \%)$, whereas the remainder had extrapulmonary TB only (13.7\%). ${ }^{(2)}$ Although the overall mortality rate from TB among Singapore residents was low at 1.2 cases per 100,000 population in 2013, ${ }^{(2}$ there are multiple studies that have shown that severe PTB requiring admission to the intensive care unit (ICU) carries a high mortality rate. ${ }^{(3-12)}$

Identified risk factors for mortality include acute respiratory distress syndrome, ${ }^{(4,5)}$ need for mechanical ventilation, ${ }^{(4)}$ HIV infection, ${ }^{(7)}$ multiple organ failure, ${ }^{(3,5)}$ consolidation on chest radiograph, ${ }^{(3,5)}$ acute renal failure, ${ }^{(4)}$ early ICU admission, ${ }^{(13)}$ Acute Physiology and Chronic Health Evaluation (APACHE) II score, ${ }^{(6)}$ Sequential Organ Failure Assessment (SOFA) score, ${ }^{(14)}$ sepsis, ${ }^{(4,6)}$ low albumin, ${ }^{(7)}$ age $^{(15)}$ and nosocomial- or ventilator-associated pneumonia. ${ }^{(4,8,13)}$ Besides these factors, treating PTB in the ICU is challenging because of poor absorption of antituberculous treatment among the critically ill and the paradoxical deterioration of TB during treatment. ${ }^{(12)}$ The most common reasons for ICU admission were acute respiratory failure and multiorgan failure. ${ }^{(12)}$
The present study aimed to identify patient characteristics and risk factors associated with in-hospital mortality among patients with PTB requiring ICU management at our hospital.

\section{METHODS}

We conducted a retrospective chart review of all patients with active PTB admitted to the ICU at Singapore General Hospital (SGH), Singapore, a tertiary care university-affiliated hospital, from January 2005 to December 2010. Data was obtained from the hospital's TB registry, which had a record of all inpatients and outpatients diagnosed with TB. The definitions of risk factors that were used in our study are listed in Box 1.

\footnotetext{
Box 1. Definitions of risk factors in the present study.

- Active pulmonary tuberculosis (PTB): smear positive for acid-fast bacilli and/or culture positive for Mycobacterium tuberculosis complex

- Immunocompromised patients: patients with HIV, active malignancy or on long-term steroids

- Acute respiratory distress syndrome: diagnosed based on the Berlin Definition ${ }^{(16)}$

- Multiorgan failure: failure of more than one organ

- Multidrug resistance: resistance to at least isoniazid and rifampicin

- Miliary tuberculosis: presence of micronodules on chest radiographs

- PTB: miliary and non-miliary parenchymal tuberculosis
} 
American Thoracic Society criteria were used to diagnose hospital-acquired pneumonia and ventilator-associated pneumonia. ${ }^{(17)}$ Patients admitted for reasons not due to PTB were excluded. Data for haemoglobin, white blood cells, creatinine, albumin, sodium, alanine aminotransferase and aspartate aminotransferase levels, APACHE II score and $\mathrm{PaO}_{2} / \mathrm{FiO}_{2}$ (partial pressure of oxygen in arterial blood/fraction of inspired oxygen) ratio was taken within the first 24 hours of ICU admission. Statistical analysis was performed using IBM SPSS Statistics version 20.0 (IBM Corp, Armonk, NY, USA). For univariate analysis, chi-square test was used for categorical variables and Student's $t$-test was used for continuous variables. For multivariate analysis, logistic regression was performed. A p-value $<0.05$ was considered to be statistically significant. All values were expressed as mean \pm standard deviation. This study was approved by SingHealth's Centralised Institutional Review Board.

\section{RESULTS}

Over the six-year study period, there were 2,155 patients with active PTB infection diagnosed at SGH, of whom 83 (3.9\%) patients were admitted to the ICU. However, eight of these patients were excluded from further analysis, as their admission was unrelated to PTB (acute abdomen, $\mathrm{n}=3$; bleeding gastrointestinal tract, $\mathrm{n}=1$; spine surgery, $\mathrm{n}=1$; atrial flutter with hypotension, $\mathrm{n}=1$; postoperative complications, $\mathrm{n}=2$ ). The remaining 75 (3.5\%) patients were eligible for analysis.

The mean age of the patients was $59.9 \pm 18.5$ years. More than half of the patients were men $(n=50,66.7 \%)$ and of Chinese ethnicity ( $n=45,60.0 \%)$. There were $12(16.0 \%)$ patients with a history of smoking. The mean body mass index was $20.4 \pm 5 \mathrm{~kg} / \mathrm{m}^{2}(\mathrm{n}=63)$ and mean APACHE II score was $22.6 \pm 7.3(\mathrm{n}=60)$.

The most common comorbidities were diabetes mellitus ( $\mathrm{n}=23,30.7 \%)$ and immunocompromised host $(\mathrm{n}=25$, $33.3 \%)$. A few ( $\mathrm{n}=4,5.3 \%)$ patients had HIV coinfection. Mechanical ventilation was required for a majority $(n=67,89.3 \%)$ of patients, for a mean duration of $8.05 \pm 14.43$ days. Mean duration of ICU stay and hospital stay were $10.23 \pm 15.8$ days and $33.7 \pm 50.7$ days, respectively. ICU mortality was $48.0 \%(n=36)$ and in-hospital mortality was $62.7 \%(n=47)$. Most $(n=60$, $80.0 \%$ ) patients were not known to have PTB at the time of medical ICU (MICU) admission. Five patients had cavitations on chest radiographs reported by radiologists. Among these patients, there were four in-hospital mortalities, two of whom died in the MICU. Table I shows a comparison of the baseline characteristics of survivors and non-survivors with active PTB requiring intensive care.

Univariate analysis identified ischaemic heart disease, low albumin, APACHE II score, disseminated intravascular coagulation, shock and multiorgan failure as being significantly associated with mortality (Table I). Multivariate analysis showed that low albumin on the day of ICU admission was the only significant independent predictor of death $(p=0.033$; Table II).

\section{DISCUSSION}

In our study, patients with active PTB requiring ICU admission had high ICU and in-hospital mortality rates of $48.0 \%$ and $62.7 \%$, respectively. These were comparable to the mortality rates (range $47 \%-65.7 \%$ ) reported from China, ${ }^{(9)}$ Taiwan, ${ }^{(8)} \mathrm{Korea}^{(5)}$ and Brazil. ${ }^{(13)}$ Other centres have reported lower mortality rates in the range of $20 \%-30 \% .{ }^{(3,4)}$ A retrospective study by Erbes et al in Germany reported ICU mortality rates of $22.4 \%$ and in-hospital mortality of $25.9 \%$, ${ }^{(4)}$ which were significantly lower than the rates observed in our study. This could be attributed to the older cohort of patients (mean age $59.9 \pm 18.5$ years vs. $47.8 \pm 17$ years) and higher percentage of patients with diabetes mellitus $(30.7 \%$ vs. $13.8 \%$ ) in our study. Although diabetes mellitus itself was not a significant independent predictor of mortality for patients with PTB admitted to the ICU, it significantly affects patient morbidity. ${ }^{(18)}$ Furthermore, patients with diabetes mellitus and TB often have atypical radiographic presentations, ${ }^{(19)}$ which could potentially result in delayed diagnosis and commencement of appropriate antituberculous therapy, thereby impacting patient outcomes. $^{(20)}$

Among the risk factors investigated, only low albumin remained predictive of mortality in patients with PTB admitted to the ICU after multivariate analysis. Previous studies have shown that lower serum albumin in the critically ill was associated with infection and increased mortality. ${ }^{(20-23)}$ Hypoalbuminaemia has been shown to be associated with increased morbidity and mortality after both non-cardiac ${ }^{(23)}$ and cardiac surgeries. ${ }^{(23)}$ In the National Veterans Affairs Surgical Risk Study, which involved 44 tertiary care veterans affairs medical centres, a decrease in serum albumin to less than $21 \mathrm{~g} / \mathrm{L}$ was associated with an increase in mortality rates, from under $1 \%$ to $29 \%$. ${ }^{(23)}$ This is not surprising because albumin plays an important role in inflammation and is also a marker of nutrition and disease. ${ }^{(24)}$ Low albumin is an early marker of protein-energy malnutrition that is associated with the stress of illness and surgeries. The mismatch of protein-energy demand and supply leads to organ dysfunction, including gastrointestinal malabsorption, impaired immune response and impaired production of plasma proteins (including albumin) in the liver. ${ }^{(24)}$ Hypoproteinaemia lowers plasma oncotic pressure, increases capillary permeability and promotes alveolar oedema. It is also associated with developing pressure ulcers in the ICU. (25)

We identified ischaemic heart disease, APACHE II score, disseminated intravascular coagulation, shock and multiorgan failure to be significantly associated with mortality on univariate analysis, but not on multivariate analysis. Multiorgan failure has been shown to be associated with mortality in a few studies. ${ }^{(3,5)}$ Although ICU scoring systems of APACHE and SOFA scores have been validated for use for predicting individual patient outcomes during ICU admissions, ${ }^{(26)}$ both of these scoring systems have only been shown to be associated with mortality in patients with PTB requiring ICU care in a few studies, ${ }^{(6,14)}$ with many others not reporting such an association. ${ }^{(4,5,13,15)}$ APACHE II scores may severely underestimate the mortality risk of patients with TB who have septic shock. ${ }^{(27)}$ In our study, the mean APACHE II score was 22.6 with predicted in-hospital mortality of $46 \%$ instead of 
Table I. Comparison of baseline characteristics of survivors and non-survivors with active pulmonary tuberculosis requiring intensive care.

\begin{tabular}{|c|c|c|c|c|}
\hline \multirow[t]{2}{*}{ Variable } & \multicolumn{3}{|c|}{ No. $(\%) /$ mean \pm SD } & \multirow[t]{2}{*}{ p-value } \\
\hline & All patients $(n=75)$ & Survivors $(n=28)$ & Non-survivors $(n=47)$ & \\
\hline Age (yr) & $59.9 \pm 18.5$ & $57.7 \pm 19.8$ & $61.3 \pm 17.5$ & 0.423 \\
\hline Male & $50(66.7)$ & $19(67.9)$ & $31(66.0)$ & 0.866 \\
\hline History of smoking & $12(16.0)$ & $6(21.4)$ & $6(12.8)$ & 0.322 \\
\hline BMI $\left(\mathrm{kg} / \mathrm{m}^{2}\right)(\mathrm{n}=63)^{*}$ & $20.4 \pm 5.0$ & $20.8 \pm 5.0$ & $20.4 \pm 1.8$ & 0.781 \\
\hline \multicolumn{5}{|l|}{ Ethnicity } \\
\hline Chinese & $45(60.0)$ & $17(60.7)$ & $28(59.7)$ & 0.922 \\
\hline Malay & $25(33.3)$ & $10(35.7)$ & $15(31.9)$ & 0.736 \\
\hline Indian & $2(2.7)$ & 0 & $2(4.3)$ & 0.526 \\
\hline Others & $3(4.0)$ & $1(3.6)$ & $2(4.3)$ & 1.000 \\
\hline \multicolumn{5}{|l|}{ Comorbidity } \\
\hline Immunocompromised host & 25 (33.3) & $11(39.3)$ & $14(29.8)$ & 0.399 \\
\hline HIV & $4(5.3)$ & $2(7.1)$ & $2(4.3)$ & 0.626 \\
\hline Diabetes mellitus & $23(30.7)$ & $6(21.4)$ & $17(36.2)$ & 0.181 \\
\hline Chronic kidney disease & $15(20.0)$ & $4(14.3)$ & $11(23.4)$ & 0.340 \\
\hline Ischaemic heart disease & $12(16.0)$ & $1(3.6)$ & $11(23.4)$ & $0.023^{+}$ \\
\hline Chronic lung disease & $5(6.7)$ & $4(14.3)$ & $1(2.1)$ & 0.061 \\
\hline Stroke & $4(5.3)$ & $3(10.7)$ & $1(2.1)$ & 0.109 \\
\hline \multicolumn{5}{|l|}{ Investigation } \\
\hline Haemoglobin $(\mathrm{g} / \mathrm{dL})$ & $9.97 \pm 2.1$ & $10.3 \pm 2.2$ & $9.7 \pm 2.0$ & 0.263 \\
\hline White blood cells $\left(\times 10^{9} / \mathrm{L}\right)$ & $11.4 \pm 7.8$ & $12.1 \pm 9.0$ & $11.0 \pm 7.2$ & 0.580 \\
\hline Creatinine $(\mu \mathrm{mol} / \mathrm{L})$ & $170 \pm 190$ & $178 \pm 228$ & $165 \pm 163$ & 0.779 \\
\hline Albumin $(\mathrm{g} / \mathrm{L})$ & $20.4 \pm 7.5$ & $24.0 \pm 7.9$ & $18.3 \pm 6.6$ & $0.001^{+}$ \\
\hline Sodium (mmol/L) & $135.1 \pm 7.1$ & $136 \pm 7.0$ & $134 \pm 7.2$ & 0.215 \\
\hline AST $(U / L)$ & $155.1 \pm 437.0$ & $57.5 \pm 50.6$ & $213.2 \pm 544.0$ & 0.058 \\
\hline ALT (U/L) & $69.3 \pm 172$ & $39.5 \pm 26$ & $87.1 \pm 216$ & 0.142 \\
\hline $\mathrm{PaO}_{2} / \mathrm{FiO}_{2}$ ratio $(\mathrm{n}=66)^{*}$ & $202 \pm 154$ & $238 \pm 160$ & $173 \pm 135$ & 0.063 \\
\hline APACHE II score $(n=60)^{*}$ & $22.6 \pm 7.3$ & $19.5 \pm 6.2$ & $23.4 \pm 7.3$ & $0.033^{+}$ \\
\hline Multidrug-resistant tuberculosis & $2(2.7)$ & $1(3.6)$ & $1(2.1)$ & 0.707 \\
\hline Extrapulmonary tuberculosis & $1(1.3)$ & 0 & $1(2.1)$ & 0.437 \\
\hline Miliary tuberculosis & $8(10.7)$ & $2(7.1)$ & $6(12.8)$ & 0.445 \\
\hline AFB smear positive & $33(44.0)$ & $8(28.6)$ & $25(53.2)$ & 0.054 \\
\hline \multicolumn{5}{|l|}{ Treatment in ICU } \\
\hline Mechanical ventilation & $67(89.3)$ & $25(89.3)$ & $42(89.4)$ & 0.774 \\
\hline Duration of mechanical ventilation (day) & $8.05 \pm 14.43$ & $7.2 \pm 11.4$ & $9.0 \pm 17.2$ & 0.578 \\
\hline Steroid use & $29(38.6)$ & $8(28.6)$ & $21(44.7)$ & 0.166 \\
\hline Dialysis & $25(33.3)$ & $6(21.4)$ & $19(40.4)$ & 0.091 \\
\hline \multicolumn{5}{|l|}{ Complication in ICU } \\
\hline Death & $36(48.0)$ & - & - & - \\
\hline Acute respiratory distress syndrome & $18(24.0)$ & $5(17.9)$ & $13(27.7)$ & 0.336 \\
\hline Ventilator-associated pneumonia & $8(10.6)$ & $6(21.4)$ & $2(4.3)$ & 0.445 \\
\hline Disseminated intravascular coagulation & $17(22.7)$ & $2(7.1)$ & $15(31.9)$ & $0.021^{+}$ \\
\hline Shock & $44(58.7)$ & $12(42.9)$ & $32(68.1)$ & $0.032^{+}$ \\
\hline Multiorgan failure & $36(48.0)$ & $7(25.0)$ & $29(61.7)$ & $0.002^{+}$ \\
\hline
\end{tabular}

*Missing data for some patients for BMI $(n=12), \mathrm{PaO}_{2} / \mathrm{FiO}_{2}$ ratio $(\mathrm{n}=9)$ and APACHE II score $(n=15)$. $+\mathrm{p}<0.05$ was considered statistically significant. AFB: acid-fast bacilli; ALT: alanine aminotransferase; APACHE: Acute Physiology and Chronic Health Evaluation; AST: aspartate aminotransferase; BMI: body mass index; HIV: human immunodeficiency virus; ICU: intensive care unit; $\mathrm{PaO}_{2} / \mathrm{FiO}_{2}$ : partial pressure of oxygen in arterial blood/fraction of inspired oxygen

the observed $62.7 \%$. Unlike acute bacterial pneumonia, which usually is diagnosed early, the symptoms of TB often precede its diagnosis by months, and the delay in initiation of appropriate anti-TB treatment may have an adverse impact on mortality independent of the severity of illness upon presentation. This is a possible explanation for the disparity seen between predicted mortality from APACHE II scores and observed in-hospital mortality. ${ }^{(27)}$ The limitations of these scoring systems made them less useful and potentially misleading for patients with PTB requiring intensive care in our study. 
Table II. Multivariate logistic regression analysis of predictors of in-hospital mortality.

\begin{tabular}{lc}
\hline Variable & p-value \\
\hline Age & 0.699 \\
Ischaemic heart disease & 0.999 \\
Low albumin & $0.033^{*}$ \\
APACHE II score & 0.722 \\
Shock & 0.821 \\
Disseminated intravascular coagulation & 0.119 \\
Multiorgan failure & 0.147 \\
\hline
\end{tabular}

${ }^{*} p<0.05$ was considered statistically significant. APACHE: Acute Physiology and Chronic Health Evaluation

Nearly $80.0 \%$ of our patients were not known to have PTB at the time of MICU admission, which could have contributed to poor patient outcome. Possible causes were delayed PTB diagnosis, delayed visit to the doctor, delayed time to treatment initiation or rapid deterioration in some patients with PTB. Due to the retrospective nature of our study, information on several pertinent factors (e.g. duration of symptoms, time to TB diagnosis, time to treatment initiation and adverse reactions from TB drugs) were not available for analysis.

Although HIV infection itself is a risk factor for mortality in the ICU, ${ }^{(28)}$ our study concurred with others ${ }^{(4,13,29)}$ in finding that HIV infection was not an independent risk factor for mortality among patients with TB in the ICU. Some authors have postulated that the short duration before death was insufficient time for HIV itself to cause mortality in patients with TB requiring ICU care, and that when TB was very severe, HIV infection was not likely to influence patient outcome any further. ${ }^{(29)}$

Various studies have identified different factors for predicting mortality in patients with active PTB requiring ICU admission, but no single factor has consistently been shown to be predictive and therefore a reflection of the different patient populations being studied. The retrospective nature and small sample size of our study may also have contributed to the lack of power, leading to a failure in detecting additional associations of significance.

In conclusion, we found that over the six-year period, 75 of $2,155(3.5 \%)$ patients with active PTB required admission to the ICU as a direct consequence of PTB. Among these patients, $67(89.3 \%)$ patients required mechanical ventilation. The in-hospital mortality in our study was $62.7 \%(n=47)$, of which 36 patients died while in the ICU, giving an ICU mortality rate of $48.0 \%$ for patients with PTB requiring ICU care. Only low serum albumin level was associated with increased mortality.

\section{REFERENCES}

1. World Health Organization. Tuberculosis. Fact Sheet No. 104. Available from: http://www.who.int/mediacentre/factsheets/fs104/en/. Accessed September 8, 2015.

2. Ministry of Health, Singapore. CommunicableDiseases Surveillancein Singapore2013. Available from: https://www.moh.gov.sg/content/moh_web/home/Publications/
Reports/2014/communicable-diseases-surveillance-in-singapore-2013.html. Accessed September 8, 2015.

3. Zahar JR, Azoulay E, Klement E, et al. Delayed treatment contributes to mortality in ICU patients with severe active pulmonary tuberculosis and acute respiratory failure. Intensive Care Med 2001; 27:513-20.

4. Erbes R, Oettel K, Raffenberg M, et al. Characteristics and outcome of patients with active pulmonary tuberculosis requiring intensive care. Eur Respir J 2006; 27:1223-8

5. Lee $\mathrm{PL}$, Jerng JS, Chang $\mathrm{YL}$, et al. Patient mortality of active pulmonary tuberculosis requiring mechanical ventilation. Eur Respir J 2003; 22:141-7.

6. Ryu YJ, Koh WJ, Kang EH, et al. Prognostic factors in pulmonary tuberculosis requiring mechanical ventilation for acute respiratory failure. Respirology 2007; 12:406-11

7. dos Santos RP, Deutschendorf C, Scheid K, Goldani LZ. In-hospital mortality of disseminated tuberculosis in patients infected with the human immunodeficiency virus. Clin Dev Immunol 2011; 2011:120278

8. Lin SM, Wang TY, Liu WT, et al. Predictive factors for mortality among non-HIV-infected patients with pulmonary tuberculosis and respiratory failure. Int J Tuberc Lung Dis 2009; 13:335-40.

9. Deng $\mathrm{W}, \mathrm{Yu} \mathrm{M}, \mathrm{Ma} \mathrm{H}$, et al. Predictors and outcome of patients with acute respiratory distress syndrome caused by miliary tuberculosis: a retrospective study in Chongqing, China. BMC Infect Dis 2012; 12:121.

10. Valade S, Raskine L, Aout $M$, et al. Tuberculosis in the intensive care unit: A retrospective descriptive cohort study with determination of a predictive fatality score. Can J Infect Dis Med Microbiol 2012; 23:173-8.

11. Kim CW, Kim SH, Lee SN, et al. Risk factors related with mortality in patient with pulmonary tuberculosis. Tuberc Respir Dis (Seoul) 2012; 73:38-47.

12. Hagan G, Nathani N. Clinical review: tuberculosis on the intensive care unit. Crit Care 2013; 17:240.

13. Silva DR, Gazzana MB, Dalcin Pde T. [Severe tuberculosis requiring ICU admission]. J Bras Pneumol 2012; 38:386-94. English, Portuguese.

14. Lee $\mathrm{K}, \mathrm{Kim} \mathrm{JH}$, Lee $\mathrm{JH}$, et al. Acute respiratory distress syndrome caused by miliary tuberculosis: a multicentre survey in South Korea. Int J Tuberc Lung Dis 2011; 15:1099-103.

15. Kim YJ, Pack KM, Jeong E, et al. Pulmonary tuberculosis with acute respiratory failure. Eur Respir J 2008; 32:1625-30.

16. ARDS Definition Task Force, Ranieri VM, Rubenfeld GD, et al. Acute respiratory distress syndrome: the Berlin Definition. JAMA 2012; 307:2526-33.

17. American Thoracic Society; Infectious Diseases Society of America. Guidelines for the management of adults with hospital-acquired, ventilator-associated, and healthcare-associated pneumonia. Am J Respir Crit Care Med 2005; 171:388-416.

18. Kapur A, Harries AD. The double burden of diabetes and tuberculosis - public health implications. Diabetes Res Clin Pract 2013; 101:10-9.

19. Tatar D, Senol G, Alptekin S, et al. Tuberculosis in diabetics: features in an endemic area. Jpn J Infect Dis 2009; 62:423-7.

20. Domínguez de Villota E, Mosquera JM, Rubio JJ, et al. Association of a low serum albumin with infection and increased mortality in critically ill patients. Intensive Care Med 1980; 7:19-22

21. Sung J, Bochicchio GV, Joshi $M$, et al. Admission serum albumin is predicitve of outcome in critically ill trauma patients. Am Surg 2004; 70:1099-102.

22. Hoeboer SH, Oudemans-van Straaten HM, Groeneveld AB. Albumin rather than $\mathrm{C}$-reactive protein may be valuable in predicting and monitoring the severity and course of acute respiratory distress syndrome in critically ill patients with or at risk for the syndrome after new onset fever. BMC Pulm Med 2015; 15:22.

23. Gibbs J, Cull W, Henderson W, et al. Preoperative serum albumin level as a predictor of operative mortality and morbidity: results from the National VA Surgical Risk Study. Arch Surg 1999; 134:36-42.

24. Lipschitz DA. Protein-energy malnutrition. Hosp Pract (Off Ed) 1988; 23:87-99.

25. Serra R, Caroleo S, Buffone G, et al. Low serum albumin level as an independent risk factor for the onset of pressure ulcers in intensive care unit patients. Int Wound J 2014; 11:550-3

26. Breslow MJ, Badawi O. Severity scoring in the critically ill: part 1--interpretation and accuracy of outcome prediction scoring systems. Chest 2012; 141:245-52.

27. Kethireddy S, Light RB, Mirzanejad Y, et al; Cooperative Antimicrobial Therapy of Septic Shock (CATSS) Database Group. Mycobacterium tuberculosis septic shock. Chest 2013; 144:474-82.

28. Le Gall JR, Lemeshow S, Saulnier F. A new Simplified Acute Physiology Score (SAPS II) based on a European/North American multicenter study. JAMA 1993; 270:2957-63

29. Lanoix JP, Gaudry S, Flicoteaux R, Ruimy R, Wolff M. Tuberculosis in the intensive care unit: a descriptive analysis in a low-burden country. Int J Tuberc Lung Dis 2014; 18:581-7. 